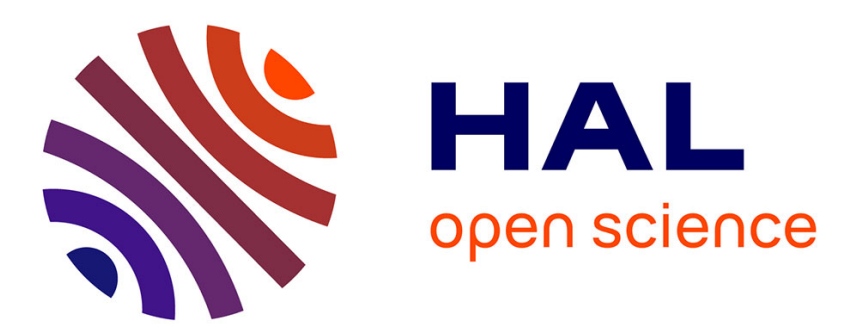

\title{
Design of orbitally stable zero dynamics for a class of nonlinear systems
}

Frédéric Grognard, Carlos Canudas de Wit

\section{To cite this version:}

Frédéric Grognard, Carlos Canudas de Wit. Design of orbitally stable zero dynamics for a class of nonlinear systems. Systems and Control Letters, 2004, 51 (2), pp.89-103. 10.1016/S0167-6911(03)002093. hal-01091542

\section{HAL Id: hal-01091542 \\ https://inria.hal.science/hal-01091542}

Submitted on 5 Dec 2014

HAL is a multi-disciplinary open access archive for the deposit and dissemination of scientific research documents, whether they are published or not. The documents may come from teaching and research institutions in France or abroad, or from public or private research centers.
L'archive ouverte pluridisciplinaire HAL, est destinée au dépôt et à la diffusion de documents scientifiques de niveau recherche, publiés ou non, émanant des établissements d'enseignement et de recherche français ou étrangers, des laboratoires publics ou privés. 


\title{
Design of orbitally stable zero dynamics for a class of nonlinear systems
}

\author{
F. Grognard ${ }^{\star}$, C. Canudas-de-Wit ${ }^{\dagger}$ \\ * INRIA Sophia-Antipolis- Projet COMORE \\ 2004 route des Lucioles, BP 93 \\ 06902 Sophia-Antipolis Cedex, France \\ frederic.grognard@sophia.inria.fr \\ † Laboratoire d'Automatique de Grenoble, ENSIEG, BP 46 , \\ 38402 Saint-Martin d'Hères Cedex, France \\ canudas@lag.ensieg.inpg.fr
}

\begin{abstract}
A method for the generation of globally attractive limit cycles for nonlinear systems is presented. It consists in designing an output that, when regulated through a suitable feedback, forces a limit cycle in the zero dynamics. Conditions are then given to ensure that a globally attractive limit cycle in the zero dynamics results in a globally attractive limit cycle in the whole system. The method is illustrated on the torque control of an induction motor.
\end{abstract}

Keywords: Limit cycles, feedback linearization, zero dynamics, induction motor.

In many applications, the natural operating mode of a control system is an oscillating one. However, the oscillations are not always present in the openloop dynamics. Therefore, it is relevant to study new control design methods forcing the internal system dynamics (or a given output) to present a prespecified limit cycle. Examples of such systems are: walking mechanisms (the full system state should behave periodically), rotating machines (the internal states, i.e. current and flux, are oscillatory if the torque output is kept constant), the synchronization of a vertically landing aircraft with the oscillation of a platform (e.g. an aircraft carrier), etc. 
There exist some published works addressing problems in this category. Under the hypothesis of the existence of a limit cycle, Hauser and Choo (see [6]) present a setup for the computation of Lyapunov functions, allowing to determinate if the given limit cycle is exponentially stable. Nevertheless no procedure is presented for the generation of the limit cycle itself. This latter problem has recently been addressed by the works of Marconi et al [9], and by Canudas de Witet al [3]. In [9], the authors deal with the problem of tracking an oscillatory signal. The addressed problem is the motion synchronization of a vertically landing aircraft with the oscillation of a platform. In [3], a feedback law that generates globally stable orbits for an underactuated inverted pendulum is presented. This later work is motivated by the walking mechanism, for which the natural operating mode is a periodic one.

In certain cases, the oscillatory internal behavior is a byproduct of an output regulation problem. An example is the torque and flux norm regulation problem for the induction motor. The linearization of these two outputs(as originally proposed by [4]), leads to an oscillating behavior in the internal dynamics. Indeed, under this particular frame, the flux and current vector asymptotically converge to a linear stable oscillation (see [1]). In [3], an output is designed and regulated such that the resulting zero dynamics of the system present a limit cycle. Evenmore, the family of outputs that is proposed allows for enough freedom such that the solutions of the zero dynamics can converge towards any prespecified closed curve.

In this paper, we will present a methodology for the design of such outputs that, when regulated through a suitable feedback, force the presence of attractive limit cycles in the zero dynamics. We consider a class of nonlinear systems having a larger number of inputs than outputs such that we have the freedom of generating new outputs. The outputs can then be decomposed into two categories: the regulated outputs, and the auxiliary outputs. Regulated outputs are the ones that reflect the main control objective (i.e. velocity or torque regulation). Auxiliary outputs are free functions of the state to be designed such that the resulting zero dynamics presents a stable limit cycle (i.e. flux).

Also we give a condition for the global attractivity of the limit cycle to be retained in the dynamics of the whole system. This result is presented in the more general setting where the limit cycle is replaced by a compact set.

This paper is structured as follows. In Section 1, we present a class of cascade systems in normal form. We first present a known result for the global stabilization of the origin of this class of systems by partial state feedback, and then we introduce our results about the global attractivity of limit cycles in such structures. In Section 2, we introduce a methodology for the design of an output that forces the system to present limit cycles. The 
system is then controlled by using input-output feedback linearization, so that the resulting system is in the form presented in Section 1. We consider the induction motor in Section 3 as an example in which we generate an attractive limit cycle by designing a suitable output using the methodology of Section 2. Finally, we give some simulations and a conclusion.

\section{Attractive limit sets in cascade systems}

In this paper, we will consider the problem of generating a limit cycle in the zero dynamics of affine nonlinear systems. In order to also have a globally attractive limit cycle in the dynamics of the complete system, some conditions need to be satisfied on the interconnection between the zero dynamics and the rest of the system, and on the stability of the limit cycle in the zero dynamics. Therefore, we analyze the nonlinear system in normal form

$$
\left\{\begin{array}{l}
\dot{z}=f(z)+\psi(z, \xi) \\
\dot{\xi}=A \xi+B u
\end{array}\right.
$$

where $\xi \in \mathbb{R}^{r}, z \in \mathbb{R}^{n-r}, u \in \mathbb{R}^{m}(m \leq n)$, and the functions $f$ and $\psi$ are locally Lipschitz continuous on their domains of definition. Function $\psi$ is such that $\psi(z, 0)=0$ for all $z$ (without loss of generality; indeed, if it were not the case, $f$ is redefined as $f(z)+\psi(z, 0)$ and $\psi$ as $\psi(z, \xi)-\psi(z, 0))$. The zero dynamics are represented by $\dot{z}=f(z)$.

Many stabilization designs are known for this particular normal form. We will present a result of [11] about the stabilization of the origin of this system by partial state feedback. This result will be needed in the proofs of the main theorems of this paper. It supposes that the interconnecting term $\psi(z, \xi)$ is linearly bounded in $z$ when $\|z\|>M$ for some $M>0$, so that finite escape time cannot occur. Also, there exists a positive semidefinite radially unbounded function $W(z)$, that decreases along the solutions of the system when $\|z\|$ is large:

Assumption 1 Suppose that there exists $M \geq 0, \eta_{1}$ and $\eta_{2}$ class $\mathcal{K}$ functions such that

$$
\|\psi(z, \xi)\| \leq \eta_{1}(\|\xi\|)\|z\|+\eta_{2}(\|\xi\|)
$$

for $\|z\| \geq M$.

Assumption 2 Suppose that there exists a positive semidefinite radially unbounded function $W(z)$ and positive constants $C$ and $M$ such that, for all $\|z\|>M$, the following holds: 
(a) $L_{f} W(z) \leq 0$;

(b) $\left\|\frac{\partial W}{\partial z}\right\|\|z\| \leq C W(z)$;

Point (b) of Assumption 2 is classically satisfied by Lyapunov functions $W(z)$ produced by converse theorems for exponentially stable systems ([8]).

Theorem 1 ([11]) Suppose that Assumptions 1 and 2 are satisfied for system (1) for which $f: \mathbb{R}^{n-r} \rightarrow \mathbb{R}^{n-r}$ and $\psi: \mathbb{R}^{n-r} \times \mathbb{R}^{r} \rightarrow \mathbb{R}^{n-r}$ are locally Lipschitz continuous functions. If $(A, B)$ is controllable, then any feedback in the form $u=k(\xi)$ guaranteeing global asymptotic stability (GAS) and local exponential stability (LES) of the origin of system $\dot{\xi}=A \xi+B k(\xi)$ yields bounded solutions for the resulting closed-loop system

$$
\left\{\begin{array}{l}
\dot{z}=f(z)+\psi(z, \xi) \\
\dot{\xi}=A \xi+B k(\xi)
\end{array}\right.
$$

Moreover, if the equilibrium $z=0$ of $\dot{z}=f(z)$ is $G A S$, then the origin of system (2) is GAS.

This theorem is proven by first showing that $W(z)$ stays bounded. Boundedness of $z$ then comes from the radial unboundedness of $W(z)$. Global stability and global attractivity follow from the Barbashin-Krasovskii principle.

\section{Almost global attractivity of a compact set}

Nonlinear systems can present many different kinds of $\omega$-limit sets other than an equilibrium point (e.g. see [5]). In this paper, we are interested in the case where one $\omega$-limit set is a limit cycle. An important property of the limit cycles is that they are compact sets; therefore, we will analyze the situation where one $\omega$-limit set is a compact set $\gamma$.

Two situations will be considered to extend Theorem 1: the case where the $z$-dynamics $(\dot{z}=f(z))$ is globally defined, and the case where the $z$ dynamics is defined everywhere except for one value of $z$. Both of these situations are considered because, in the context of partially linearizable systems, system (1) is often the normal form corresponding to a given affine system with $m$ inputs and $m$ outputs. The second situation may arise when the change of coordinates leading to this normal form is not a global diffeomorphism. However, we want attractive compact sets in $\dot{z}=f(z)$ to be captured by system (1) whether there are singularities or not. To cover both cases, the notion of almost global attractivity of a compact set is used. 
Definition 1 A compact set $\gamma$ is "almost globally attractive" for the dynam$i c s$

$$
\dot{x}=f(x)
$$

with $x \in \mathbb{R}^{n}$, if it is attractive with basin of attraction containing the whole state space minus a set of Lebesgue measure zero.

In dimension 2, the simple situation where a limit cycle $\gamma$ attracts every solution except those starting at the mandatory equilibrium inside the area circumscribed by $\gamma$ fits into Definition 1: $\gamma$ is almost globally attractive because the equilibrium is of Lebesgue measure zero.

\subsection{Globally defined dynamics}

In this section, we consider the special case where the union of the $\omega$-limit sets of (3) is made of an almost globally attractive compact set $\gamma$ and an equilibrium $\bar{x}$ such that $\gamma$ and $\bar{x}$ are disjoint.

Remark 1 Note that $\bar{x}$ is unstable. Indeed, if $\bar{x}$ is stable without being asymptotically stable, there are solution whose $\omega$-limit set is neither $\bar{x}$ nor $\gamma$. On the other hand if $\bar{x}$ is asymptotically stable, the regions of attraction of $\bar{x}$ and $\gamma$ are open, non empty, connected sets (see [8]). Therefore, $\mathbb{R}^{n}$ needs to be covered by two disjoint, non empty, open sets, which is a contradiction. Therefore $\bar{x}$ is unstable.

If we consider the existence of an almost globally attractive compact set $\gamma$ in the $z$-dynamics, the next theorem gives conditions for the almost globally attractivity of $\gamma$ to translate into almost global attractivity of $\Gamma \equiv\{(z, \xi) \in$ $\mathbb{R}^{n} \mid z \in \gamma$ and $\left.\xi=0\right\}$ in the interconnected system.

Theorem 2 Suppose that Assumptions 1 and 2 are satisfied for system (1) for which $f: \mathbb{R}^{n-r} \rightarrow \mathbb{R}^{n-r}$ and $\psi: \mathbb{R}^{n-r} \times \mathbb{R}^{r} \rightarrow \mathbb{R}^{n-r}$ are locally Lipschitz continuous functions. Let the only invariant sets of $\dot{z}=f(z)$ be $z=0$ and $\gamma$, respectively an equilibrium and an almost globally attractive compact set $(0 \notin \gamma)$. If $(A, B)$ is controllable, then any feedback in the form $u=k(\xi)$ guaranteeing that the origin of $\dot{\xi}=A \xi+B k(\xi)$ is $G A S$-LES yields

(i) convergence of the solutions of

$$
\left\{\begin{array}{l}
\dot{z}=f(z)+\psi(z, \xi) \\
\dot{\xi}=A \xi+B k(\xi)
\end{array}\right.
$$

to the unstable equilibrium $(z, \xi)=(0,0)$ or to the compact set $\Gamma$. 
(ii) If the set of initial conditions of solutions converging to $(0,0)$ is of Lebesgue measure zero, the compact set $\Gamma$ is almost globally attractive.

(iii) If the equilibrium of $\dot{z}=f(z)$ is hyperbolic, and if the global stable manifold of the origin $(0,0)$ is a manifold whose dimension is globally defined and constant, the compact set $\Gamma$ is almost globally attractive.

(iv) If $\gamma$ is an exponentially stable periodic orbit for $\dot{z}=f(z)$, then $\Gamma$ is an exponentially stable periodic orbit for (4).

Proof: Boundedness of the solutions directly comes from Theorem 1. Indeed, Assumptions 1 and 2 are still satisfied.

Every solution of (4) converges to the set $E=\left\{(z, \xi) \in \mathbb{R}^{n} \mid \xi=0\right\}$. LaSalle's invariance principle then asserts that every bounded solution converges to the largest invariant set of $E$. This set is defined by the largest invariant set of $\dot{z}=f(z)$ : the origin $z=0$ and the compact set $\gamma$. Because every solution of (4) is bounded, every solution either converges towards the origin $(0,0)$ or towards the compact set $\Gamma$. Moreover, Remark 1 implies that $z=0$ is an unstable equilibrium in the $z$-dynamics. The fact that $(z, \xi)=(0,0)$ is unstable directly follows, which shows (i).

The basin of attraction of the origin $(0,0)$ is the set of initial conditions of solutions not converging to $\Gamma$. Because it is of Lebesgue measure zero, the compact set $\Gamma$ is almost globally attractive, which shows (ii).

If the origin $z=0$ is a hyperbolic fixed point for $\dot{z}=f(z)$, the set of eigenvalues of the Jacobian linearization $\frac{\partial f}{\partial z}(0)$ contains $n_{u} \geq 1$ (resp. $n_{s} \leq$ $n-r-1)$ eigenvalues with positive (resp. negative) real parts $\left(n_{s}+n_{u}=n-r\right)$.

Since the origin $\xi=0$ is locally exponentially stable for $\dot{\xi}=A \xi+B k(\xi)$, the Jacobian linearization $A+B \frac{\partial k}{\partial \xi}(0)$ has $r$ eigenvalues with negative real parts. The Jacobian linearization of the complete system (4) is then

$$
\mathcal{J}=\left(\begin{array}{cc}
\frac{\partial f}{\partial z}(0)+\frac{\partial \psi}{\partial z}(0,0) & \frac{\partial \psi}{\partial \xi}(0,0) \\
0 & A+B \frac{\partial k}{\partial \xi}(0)
\end{array}\right)
$$

Because $\psi(z, 0)=0$ for all $z, \frac{\partial \psi}{\partial z}(0,0)=0$. Therefore, the eigenvalues of $\mathcal{J}$ are those of $\frac{\partial f}{\partial z}(0)$ and of $A+B \frac{\partial k}{\partial \xi}(0)$. $\mathcal{J}$ has then $n_{s}+r \leq n-1$ eigenvalues with negative real parts and $n_{u} \geq 1$ eigenvalues with positive real parts. The stable manifold theorem for a fixed point (see [5]) then states that there exists a local stable manifold $\mathcal{M}_{s}$ of dimension $n_{s}+r$ and a local unstable manifold $\mathcal{M}_{u}$ of dimension $n_{u}$ at the origin. If the dimension of the global stable manifold is globally defined and constant, this global stable manifold also has a dimension $n_{s}+r$. Therefore the set of initial conditions of solutions 
that converge to the origin $(0,0)$ (and not to $\Gamma$ ) lies in a manifold of dimension smaller or equal to $n-1$. Because a manifold of dimension smaller or equal to $n-1$ is of Lebesgue measure zero in $\mathbb{R}^{n}$, the compact set $\Gamma$ is almost globally attractive, which proves (iii).

Exponential stability of $\gamma$ implies the existence of a Lyapunov function $V_{1}(z)$ that is decreasing along the solutions of $\dot{z}=f(z)$. This function satisfies

$$
L_{f} V_{1} \leq-k_{1}\|z\|_{\gamma}^{2}
$$

where $\|z\|_{\gamma}=\inf _{y \in \gamma}\|z-y\|([6])$. On the other hand, a similar Lyapunov function $V_{2}(\xi)$ can be found for the $\xi$ subsystem $\left(m_{1}\|\xi\|^{2} \leq V_{2}(\xi) \leq\right.$ $m_{2}\|\xi\|^{2}, L_{A \xi+B k(\xi)} V_{2} \leq-m_{3}\|\xi\|^{2}$ ). As in [8], we then define the Lyapunov function:

$$
V(z, \xi)=V_{1}(z)+2 k \sqrt{V_{2}(\xi)}
$$

with $k>0$, whose derivative is

$$
\begin{aligned}
\dot{V} & =L_{f} V_{1}(z)+L_{\psi} V_{1}(z)+k \frac{L_{A \xi+B k(\xi)} V_{2}}{\sqrt{V_{2}}} \\
& \leq-k_{1}\|z\|_{\gamma}^{2}+\frac{d V_{1}}{d z} \psi(z, \xi)-k m_{3} \frac{\|\xi\|^{2}}{\sqrt{V_{2}}} \\
& \leq-k_{1}\|z\|_{\gamma}^{2}-\frac{k m_{3}}{m_{1}}\|\xi\|+\frac{d V_{1}}{d z} \psi(z, \xi) \\
& \leq-k_{1}\|z\|_{\gamma}^{2}-\frac{k m_{3}}{m_{1}}\|\xi\|+M\|\xi\|
\end{aligned}
$$

where the last inequality is valid in a small neighborhood of $\Gamma$ because $\frac{d V_{1}}{d z} \psi(z, \xi)$ is continuously differentiable. Taking $k>\frac{m_{1} M}{m_{3}}$ ensures negative definiteness of the derivative of $V$. The closed orbit $\Gamma$ is therefore exponentially stable. This proves (iv).

The origin of the complete closed-loop system behaves like a saddle point: it is attractive in some directions and repulsive in others. This is illustrated in Figure 1, where $\gamma$ is a limit cycle, $n=3$, and $r=1$. In this figure, the $z$ system is of dimension 2 with an almost globally attractive limit cycle $\gamma$. In order to clarify the figure, we suppose that the stable manifold $\mathcal{M}_{s}$ of the origin is the $\xi$ axis. Therefore, every solution starting on that axis converge to the origin $(0,0,0)^{T}$. All the other solutions converge to the limit cycle because the origin is only attractive in the $\xi$ direction.

\subsection{Almost globally defined dynamics}

As previously stated, we will consider another situation where almost global attractivity of a compact set is necessary: the case where the union of the 


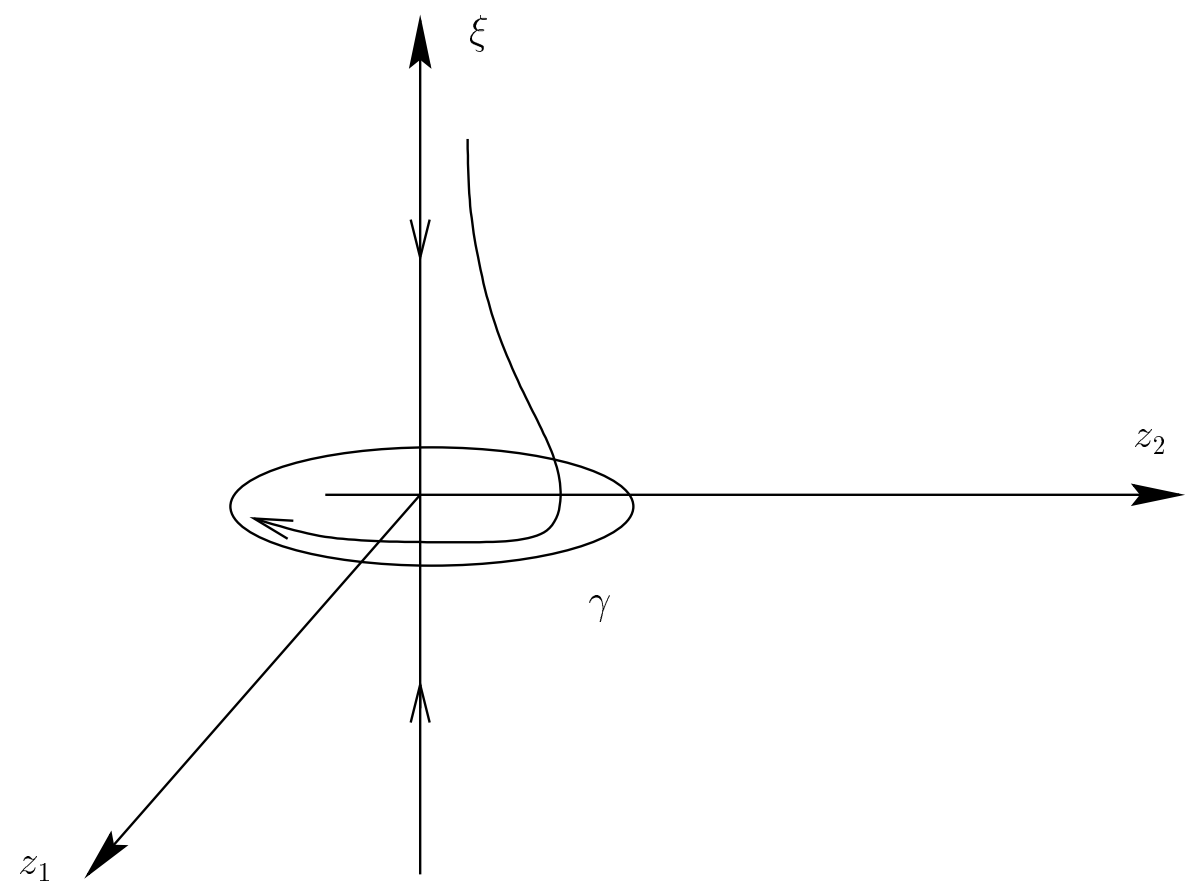

Figure 1: Almost global attractivity of a limit cycle. Solutions starting on the $\xi$ axis converge to the origin. All others converge to $\gamma$.

$\omega$-limit sets of the $z$ dynamics only consists of an almost globally attractive compact set $\gamma$. Instead of the $z$-dynamics presenting an equilibrium at $z=0$, we now consider that these dynamics are not defined at $z=0^{1}$. However, we must now impose that solutions of the closed-loop $(z, \xi)$ never go through $z=0$. This is ensured by assuming that the origin of the $z$ subsystem is repulsive in the interconnected system: this can be obtained by having $\dot{W}>0$ when $z$ is small. Therefore we now assume

Assumption 3 For every compact set $\mathcal{C}$ of $\mathbb{R}^{r}$, there exists $\delta>0$ such that for any vector $z \neq 0$ and any vector $\xi$ satisfying

$0 \leq W(z) \leq \delta$ and $\xi \in \mathcal{C}$

where the function $W$ is from Assumption 2, the following inequality

$$
L_{f(z)+\psi(z, \xi)} W(z)=\frac{\partial W(z)}{\partial z}(f(z)+\psi(z, \xi)>0
$$

\footnotetext{
${ }^{1}$ This problem may come from the potential state singularities that arise while transforming a nonlinear system into the normal form (1). An example is given in Section 3
} 
holds.

This assumption yields solutions which stay away from the axis $z=0$. Indeed, when the $\xi$ subsystem is stabilized, $\xi$ evolves in a compact set $\mathcal{C}$, so that $L_{f(z)+\psi(z, \xi)} W(z)>0$ when $z$ is close to 0 . Assumption 3 can be satisfied if $L_{f(z)} W(z)$ tends to $+\infty$ as $z$ goes to zero (the origin is "strongly" repulsive for $\dot{z}=f(z))$ while, for any fixed $\xi, L_{\psi(z, \xi)} W(z)$ stays bounded as $z$ goes to zero (which is coherent with the linear boundedness of $\psi$ with respect to $z$ that is imposed in Assumption 1). An example of such a function is given in Section 3.

Theorem 3 Suppose that Assumptions 1, 2, and 3 are satisfied for system (1) with $f: \mathbb{R}_{0}^{n-r} \rightarrow \mathbb{R}^{n-r}$ and $\psi: \mathbb{R}_{0}^{n-r} \times \mathbb{R}^{r} \rightarrow \mathbb{R}^{n-r}$, locally Lipschitz continuous functions. Let the only invariant of $\dot{z}=f(z)$ be $\gamma(0 \notin \gamma)$, an almost globally attractive compact set. If $(A, B)$ is controllable, then any feedback in the form $u=k(\xi)$ guaranteeing that the origin of $\dot{\xi}=A \xi+B k(\xi)$ is GAS-LES yields convergence of the solutions of

$$
\left\{\begin{array}{l}
\dot{z}=f(z)+\psi(z, \xi) \\
\dot{\xi}=A \xi+B k(\xi)
\end{array}\right.
$$

to the compact set $\Gamma$ (if the initial condition $(z(0), \xi(0))$ is such that $z(0) \neq 0)$. Therefore, $\Gamma$ is almost globally attractive.

Proof: Boundedness of the solutions directly comes from Theorem 1 .

The stability of the controlled $\xi$ subsystem implies that $\xi(t)$ is bounded. This boundedness implies that $\xi(t)$ lies in a compact set $\mathcal{C}$ for all $t \geq 0$. The proof of the impossibility of $z$ to go through or converge towards $z=0$ is now made considering only the system $\dot{z}=f(z)+\psi(z, \xi)$, with $\xi$ a time-varying signal belonging to $\mathcal{C}$ :

Assumption 3 ensures that there exists $\delta>0$, defining a bounded set $\Delta=\left\{z \in \mathbb{R}_{0}^{n-r} \mid 0 \leq W(z) \leq \delta\right\}$, such that $\dot{W}(z)=L_{f(z)+\psi(z, \xi)} W(z)>0$ for $z$ inside $\Delta$ and $\xi$ inside $\mathcal{C}$.

This implies:

(a) the positive invariance of $\mathbb{R}^{n-r} \backslash \Delta$, so that $z(t)$ never enters $\Delta$ (if $z(0) \notin \Delta)$;

(b) if $z(0) \in \Delta$, so that there exists $0 \leq \delta_{0} \leq \delta$ such that $W(z(0))=\delta_{0}$, we have $W(z(t))>\delta_{0}$ for all $t>0$.

Both those points show that $z(t)$ cannot go through or converge towards $z=0$. The set $\left\{(z, \xi) \in \mathbb{R}^{n} \mid z \neq 0\right\}$ is then a positive invariant set of $(5)$. 
Every solution of $(5)$, with $z(0) \neq 0$, then converges to the set $E=$ $\left\{(z, \xi) \in \mathbb{R}^{n} \mid \xi=0\right.$ and $\left.z \neq 0\right\}$. LaSalle's invariance principle states that every bounded solution converges to the largest invariant set of $E$. This set is defined by the largest invariant set of the $z$-dynamics: the compact set $\gamma$. Every solution of $(5)$, with $z(0) \neq 0$, then converges to the compact set $\Gamma$.

The set $\{(z, \xi) \mid z=0\}$ is of Lebesgue measure zero in $\mathbb{R}^{n}$. Therefore the set of initial conditions that produce undefined solutions, so that they do not converge towards the compact set, is of Lebesgue measure zero. $\Gamma$ is an almost globally attractive compact set.

This proof also shows that the absence of definition of the vector field in $z=0$ is not a problem for the definition of solutions. Indeed, whether the initials conditions are inside or outside $\Delta$, there exists $\delta_{0}$ such that $W(z)>\delta_{0}$ along the corresponding solution. The state $z$ stays inside an open subset of $\mathbb{R}^{n-r}$ inside which the vector field is locally Lipschitz continuous.

The picture of the complete closed-loop system is similar to that of Figure 1 , the only difference being the absence of definition of solutions on the $\xi$ axis instead of the convergence to the origin.

\section{Limit cycles}

In the particular case where the compact limit set $\gamma$ is an almost globally attractive limit cycle for the $z$-dynamics, it remains to show that the almost globally attractive compact limit set $\Gamma$ is a limit cycle for the complete dynamics. This fact is based on the definition of a limit cycle in forward time $([8])$ :

Definition 2 A limit cycle is a closed orbit $\gamma$ such that $\gamma$ is the positive limit set of a positive semiorbit $\gamma^{+}(y)$ for some $y \notin \gamma$.

Because $\gamma$ is a limit cycle for $\dot{z}=f(z)$, there exists $z_{0}$ such that $\gamma$ is the positive limit set of a positive semiorbit with initial condition $z_{0}$.

In the complete dynamics, $\Gamma$ is obviously a closed orbit (because the dynamics on $\Gamma$ are $\dot{z}=f(z), \dot{\xi}=0)$. It then suffices to take $\left(z_{0}, 0\right)$ as initial condition of a positive semiorbit, and $\Gamma$ is the positive limit set of this semiorbit. The almost globally attractive compact set $\Gamma$ is then a limit cycle.

\section{Design of orbitally stable zero dynamics}

In this section, we consider the main problem of this paper: the generation of limit cycles through the design of orbitally stable zero dynamics. Therefore, 
we consider an affine nonlinear system with more inputs than outputs:

$$
\left\{\begin{array}{l}
\dot{x}=f_{0}(x)+g(x) u \\
y=h(x)
\end{array}\right.
$$

with $x \in \mathbb{R}^{n}, y=\left(y_{1}, \cdots, y_{m-p}\right)^{T}=\left(h_{1}(x), \cdots, h_{m-p}(x)\right)^{T} \in \mathbb{R}^{m-p}, u=$ $\left(u_{1}, \cdots, u_{m}\right)^{T} \in \mathbb{R}^{m}(m<n, 0<p \leq m)$. The functions $f_{0}: \mathbb{R}^{n} \rightarrow \mathbb{R}^{n}$, $g: \mathbb{R}^{n} \rightarrow \mathbb{R}^{m}$, and $h: \mathbb{R}^{n} \rightarrow \mathbb{R}^{m-p}$ are smooth.

Example 1 In this section, we will illustrate our design method on the following system in the form (6):

$$
\left\{\begin{array}{l}
\dot{x}_{1}=x_{2} x_{3}+u_{1} \\
\dot{x}_{2}=-x_{1} x_{3}+x_{2} u_{2} \\
\dot{x}_{3}=x_{1} x_{2}+x_{3} u_{2} \\
y_{1}=x_{1}-\bar{x}_{1}
\end{array}\right.
$$

If $y_{1}$ is regulated, and the second input $u_{2}$ is not used $\left(u_{2}=0\right)$, the $\left(x_{2}, x_{3}\right)$ subsystem behaves like an harmonic oscillator with angular velocity $\bar{x}_{1}$. There is no attractive limit cycle. The oscillations are neutrally stable.

The excess of inputs with respect to the outputs leaves the possibility of designing $p$ supplementary outputs. Those $p$ outputs will be designed so that the zero dynamics of the system presents a limit cycle; as a consequence, the regulation of the outputs induces a limit cycle in the dynamics of the control system. The outputs can be divided into two classes: the regulated outputs: those are given by $y=h(x)$ (the regulation of these outputs is required for the solution of the given control problem); the auxiliary outputs: those are designed to generate a limit cycle in the dynamics. The control approach that will be used includes an input-output linearization by feedback ([7]).

Linearization by feedback The design of zero dynamics presenting an almost globally attractive limit cycle requires the construction of $p$ new outputs $\left(y_{m-p+1}, \cdots, y_{m}\right)^{T}=\left(h_{m-p+1}(x), \cdots, h_{m}(x)\right)^{T}$. The addition of these new outputs to $y$ allows for the definition of relative degree of the output $\left(y_{1}, \cdots, y_{m}\right)^{T}$ as integers $\left(r_{1}, \cdots, r_{m}\right)$ that satisfy

$$
L_{g_{j}} L_{f_{0}}^{k} h_{i}(x)=0
$$

for $1 \leq i, j \leq m$, and $0 \leq k \leq r_{i}-2$. Also, the matrix

$$
R(x)=\left(\begin{array}{ccc}
L_{g_{1}} L_{f_{0}}^{r_{1}-1} h_{1}(x) & \cdots & L_{g_{m}} L_{f_{0}}^{r_{1}-1} h_{1}(x) \\
\vdots & & \vdots \\
L_{g_{1}} L_{f_{0}-1}^{r_{m}-1} h_{m-1}(x) & \cdots & L_{g_{m}} L_{f_{0}-1}^{r_{m-1}} h_{m-1}(x) \\
L_{g_{1}} L_{f_{0}}^{r_{m}-1} h_{m}(x) & \cdots & L_{g_{m}} L_{f_{0}}^{r_{m}-1} h_{m}(x)
\end{array}\right)
$$


must be regular. If the relative degree is globally defined and constant, the linearization by feedback of the relation

$$
\left(\begin{array}{c}
y_{1}^{\left(r_{1}\right)} \\
\vdots \\
y_{m-1}^{\left(r_{m-1}\right)} \\
y_{m}^{\left(r_{m}\right)}
\end{array}\right)=\left(\begin{array}{c}
L_{f_{0}}^{\left(r_{1}\right)} h_{1}(x) \\
\vdots \\
L_{\left.f_{0}-1\right)}^{\left(r_{m-1}\right)} h_{m-1}(x) \\
L_{f_{0}}^{\left(r_{m}\right)} h_{m}(x)
\end{array}\right)+R(x)\left(\begin{array}{c}
u_{1} \\
\vdots \\
u_{m}
\end{array}\right)=\alpha(x)+R(x) u
$$

can be achieved by defining $u=-R^{-1}(x)(\alpha(x)+v)$. This input linearizes the system from the input $v$ to the output $y$. If there exists a global diffeomorphism that globally puts the system in normal form:

$$
\begin{cases}\dot{z}^{\left(r_{1}\right)} & =f(z)+\psi\left(z, y_{1}, \cdots, y_{1}^{\left(r_{1}-1\right)}, \cdots, y_{m}, \cdots, y_{m}^{\left(r_{m}-1\right)}\right) \\ y_{1} & \vdots \\ y_{m}^{\left(r_{m}\right)} & =v_{m}\end{cases}
$$

where $\psi(z, 0)=0$, then a choice of $v(\xi)=v\left(y_{1}, \cdots, y_{1}^{\left(r_{1}-1\right)}, \cdots, y_{m}, \cdots, y_{m}^{\left(r_{m}-1\right)}\right)$ can easily be made so that the origin of the $\xi$ subsystem is globally asymptotically and locally exponentially stable. The existence of an almost globally attractive limit cycle for the complete control system then depends on the dynamics of

$$
\dot{z}=f(z)
$$

and on the verification of the different hypotheses of Theorems 2 and 3 .

Choice of $\left(y_{m-p+1}, \cdots, y_{m}\right)^{T}=\left(h_{m-p+1}(x), \cdots, h_{m}(x)\right)^{T} \quad$ In order to facilitate the analysis of the presence of a limit cycle, $z$ should be of dimension 2. Because the dimension of the system (10) is $n$ and the $\xi$ subsystem is of dimension $\sum_{i=1}^{m} r_{i}$, this sum $\sum_{i=1}^{m} r_{i}$ should be equal to $n-2$. For system (7), an output of relative degree 0 should be designed because the dimension of the system is 3 and the relative degree of $y_{1}$ is 1 .

Particular case When $p=1$, only one auxiliary output can be designed. Its relative degree should be $n-2-\sum_{i=1}^{m-1} r_{i}$.

A practical way of choosing this output consists in picking two state variables $\left(x_{a}, x_{b}\right)$ that are independent of the existing outputs and their derivatives (up to their relative degree minus one). A positive definite function $W\left(x_{a}, x_{b}\right)$ is then built and a level $W\left(x_{a}, x_{b}\right)=L$, which represents a closed 
curve, is chosen. A limit cycle is imposed on this curve by forcing the dynamics

$$
\dot{W}\left(x_{a}, x_{b}\right)=-\nu\left(W\left(x_{a}, x_{b}\right)-L\right)
$$

to be satisfied. Function $\nu$ is picked so that $W$ globally converges to $L$. The dynamics (12) are then forced by picking the last output as

$$
y_{m}=h_{m}(x)=\dot{W}\left(x_{a}, x_{b}\right)+\nu\left(W\left(x_{a}, x_{b}\right)-L\right)
$$

For $y_{m}$ to have a relative degree $n-2-\sum_{i=1}^{m-1} r_{i}$, the function $W\left(x_{a}, x_{b}\right)$ should have a relative degree $n-1-\sum_{i=1}^{m-1} r_{i}$. If this output results in zero dynamics of dimension 2, and if these dynamics can be expressed in the $\left(x_{a}, x_{b}\right)$ plane, these dynamics must satisfy (12), which forces a limit cycle inside the $\left(x_{a}, x_{b}\right)$ plane. A limit cycle must then be present in the zero dynamics and in the complete system.

Example 1 (continued) We can take $\left(x_{a}, x_{b}\right)=\left(x_{2}, x_{3}\right), W\left(x_{2}, x_{3}\right)=$ $\frac{x_{2}^{2}+x_{3}^{2}}{2}$, and $\nu(s)=2 k s$ (with $k>0$ ). This result in

$$
y_{2}=\left(x_{2}^{2}+x_{3}^{2}\right) u_{2}+k\left(x_{2}^{2}+x_{3}^{2}-L\right)
$$

The output $y_{2}$ can be set to 0 by fixing $u_{2}$ as long as $\left(x_{2}^{2}+x_{3}^{2}\right)>0$. The $y_{1}$ dynamics are fixed by $u_{1}$. The linear part of the system then resumes to

$$
\dot{y}_{1}=u_{1}
$$

The remaining dynamics can be expressed with the $\left(x_{2}, x_{3}\right)$ states by replacing $u_{2}$ in $(7)$ by the value imposed by $y_{2}=0$. It comes to

$$
\begin{aligned}
& \dot{x}_{2}=-\bar{x}_{1} x_{3}+\frac{-k x_{2}\left(x_{2}^{2}+x_{3}^{2}-L\right)}{x_{2}^{2}+x_{3}^{2}}-y_{1} x_{3} \\
& \dot{x}_{3}=\bar{x}_{1} x_{2}+\frac{-k x_{3}\left(x_{2}^{2}+x_{3}^{2}-L\right)}{x_{2}^{2}+x_{3}^{2}}+y_{1} x_{2}
\end{aligned}
$$

The first term of $\dot{x}_{2}$ and $\dot{x}_{3}$ induces the oscillation (with an angular velocity $\bar{x}_{1}$ ), the second term induces the attractivity of the level $2 L$ of $W$, and the third term is the interconnecting term with the linear part. A limit cycle is present if $L>0$ and $\bar{x}_{1} \neq 0$. In order to prove almost global attractivity of the limit cycle in the normal form (13)-(14) of system (7), Theorem 3 should be used, because the dynamics are not defined everywhere.

This method is illustrated on the following case study. 


\section{The induction motor}

To illustrate the previously proposed methodology, we consider the case of velocity control in an induction motor. Let a fifth-order model ${ }^{2}$ of the motor in stator $(\alpha-\beta)$-coordinates : the state vector $x$ includes the coordinates of the stator current $\left(I_{\alpha}\right.$ and $\left.I_{\beta}\right)$, the coordinates of the rotor flux ( $\phi_{\alpha}$ and $\left.\phi_{\beta}\right)$, as well as the mechanical angular velocity $\omega$. The input vector is the two-dimensional stator voltage. The Park's model of the induction motor is:

$$
\left\{\begin{array}{l}
\dot{I}_{\alpha}=-\zeta I_{\alpha}+\eta\left[a \phi_{\alpha}+v \omega \phi_{\beta}\right]+\frac{1}{\sigma L_{s}} U_{\alpha} \\
\dot{I}_{\beta}=-\zeta I_{\beta}+\eta\left[a \phi_{\beta}-v \omega \phi_{\alpha}\right]+\frac{1}{\sigma L_{s}} U_{\beta} \\
\dot{\phi}_{\alpha}=-a \phi_{\alpha}-v \omega \phi_{\beta}+b I_{\alpha} \\
\dot{\phi}_{\beta}=-a \phi_{\beta}+v \omega \phi_{\alpha}+b I_{\beta} \\
J \dot{\omega}=c I_{\beta} \phi_{\alpha}-c I_{\alpha} \phi_{\beta}
\end{array}\right.
$$

where $\zeta, \eta, \sigma, a, b$, and $c$ are constants depending on the electrical parameters of the motor, $L_{s}$ is the stator inductance, and $v$ is the number of pair of poles ([10]). $\tau_{e}=c I_{\beta} \phi_{\alpha}-c I_{\alpha} \phi_{\beta}$ is the electrical torque produced by the motor.

The natural primary control design goal is to regulate the motor rotational velocity (or position), to a target velocity $\omega_{0}$. This objective can be formulated by defining the primary output $y_{1}$ as

$$
y_{1}=\omega-\omega_{0}
$$

and finding a linearizing feedback for such an output. Since the control vector is of dimension two, it is still possible to define another (auxiliary) output $y_{2}$ that can also be regulated according to secondary goals. If the second output is chosen to be the flux norm,i.e.

$$
y_{2}=\|\phi\|
$$

so that its regulation preserves the machine operation under nominal conditions, it has already been proven that there exists static linearizing feedback that linearizes the output vector $y=\left(y_{1}, y_{2}\right)$, see [4].

In the context of field oriented control, this auxiliary output is typically regulated to a constant value. For constant motor velocity operation condition, this operation mode leads to a flux vector that rotates with constant frequency, thus to a behavior that is oscillatory. This results also in an oscillatory behavior of the zero dynamics.

\footnotetext{
${ }^{2}$ For simplicity of the presentation, we have assumed absence of load. This allows us to work with a model of smaller dimension.
} 
Nevertheless it is possible to track any arbitrary trajectory. In [2], the authors have proposed to control $y_{2}$ such as to minimize the internal motor energy.

This example will show that it is possible to define auxiliary output $y_{2}$, having a more general structure, such that the motion of the internal dynamics described by the flux trajectories (not their norm) presents a possible family of limit cycles.

For this we will apply the method of the previous section: the output $y_{1}$ is given and has a relative degree 2 . Therefore, $\xi_{0}=\left(y_{1}, \dot{y}_{1}\right)=\left(\omega-\omega_{0}, c\left(I_{\beta} \phi_{\alpha}-\right.\right.$ $\left.I_{\alpha} \phi_{\beta}\right)$. The states $\left(x_{a}, x_{b}\right)$ should be chosen independent of $\xi_{0}$. Like in [4], we choose $\left(x_{a}, x_{b}\right)=\left(\phi_{\alpha}, \phi_{\beta}\right)$.

As suggested in the previous section, we consider an auxiliary output in the form

$$
y_{2}=\dot{W}\left(\phi_{\alpha}, \phi_{\beta}, \theta\right)+\nu\left(W\left(\phi_{\alpha}, \phi_{\beta}, \theta\right)-L\right)
$$

where $W\left(\phi_{\alpha}, \phi_{\beta}, \theta\right)$ is a positive definite, radially unbounded function. $\theta$ is a parameter influencing the shape of the function. The positive scalar $L$ is chosen such that the level set $W\left(\phi_{\alpha}, \phi_{\beta}, \theta\right)=L$ characterizes a closed curve in the state-space $\left(\phi_{\alpha}, \phi_{\beta}\right) . \nu(s)$ is a scalar Lipschitz function, which has its image inside the first and third quadrant, with $\lim _{s \rightarrow-L} \nu(s)=-\infty$ (Figure 2).

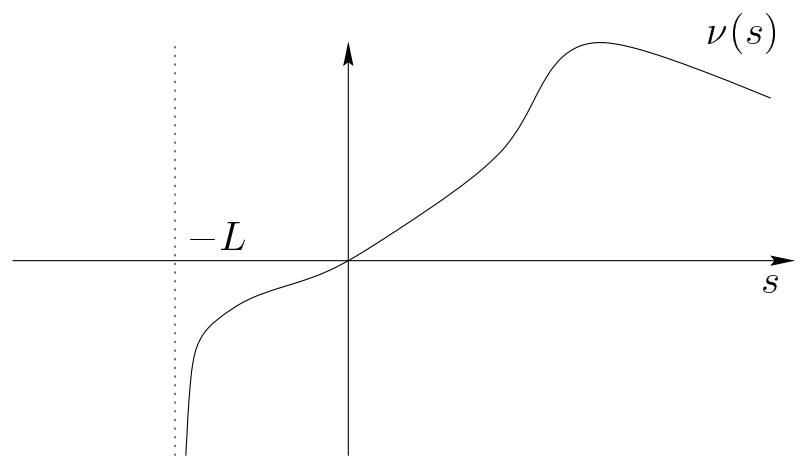

Figure 2: The function $\nu$

The main differences with [4] are that $W$ does not need to be quadratic, and the fact that the relative degree of $y_{2}$ is 1 (compared to 2 when $y_{2}$ is the norm of the flux), which increases the dimension of the zero dynamics, making more explicit the presence of a limit cycle. The expression $\dot{W}\left(\phi_{\alpha}, \phi_{\beta}, \theta\right)$ can be written as an algebraic function of the state $x$ of our system (through the expressions $\dot{\phi}_{\alpha}$ and $\dot{\phi}_{\beta}$ ). 
The induction motor is then a MIMO system with two inputs

$$
U_{\alpha} \text { and } U_{\beta}
$$

and two outputs

$$
\left\{\begin{aligned}
y_{1}= & \omega-\omega_{0} \\
y_{2}= & \frac{\partial W}{\partial \phi_{\alpha}}\left(-a \phi_{\alpha}-v \omega \phi_{\beta}+b I_{\alpha}\right) \\
& +\frac{\partial W}{\partial \phi_{\beta}}\left(-a \phi_{\beta}+v \omega \phi_{\alpha}+b I_{\beta}\right)+\nu\left(W\left(\phi_{\alpha}, \phi_{\beta}, \theta\right)-L\right)
\end{aligned}\right.
$$

The relative degree of this MIMO system is $\{2,1\}$ because $u$ first appears in the second and first derivative of $y_{1}$ and $y_{2}$, respectively: this implies that the dimension of the zero dynamics is 2 . In order to partially linearize system (15) by feedback, the matrix

$$
R(x)=\frac{1}{\sigma L_{s}}\left(\begin{array}{cc}
-c \phi_{\beta} & c \phi_{\alpha} \\
b \frac{\partial W}{\partial \phi_{\alpha}} & b \frac{\partial W}{\partial \phi_{\beta}}
\end{array}\right)
$$

of (9) needs to be globally regular because a feedback in the form $u=$ $-R^{-1}(x)(\alpha(x)+v)$ must be applied. The determinant of this matrix is $-\frac{c b}{\sigma^{2} L_{s}^{2}}\left(\frac{\partial W}{\partial \phi_{\alpha}} \phi_{\alpha}+\frac{\partial W}{\partial \phi_{\beta}} \phi_{\beta}\right)$. Therefore, the input-output linearization is globally achievable if and only if

$$
\frac{\partial W}{\partial \phi_{\alpha}} \phi_{\alpha}+\frac{\partial W}{\partial \phi_{\beta}} \phi_{\beta} \neq 0
$$

for all $\left(\phi_{\alpha}, \phi_{\beta}\right)$. Obviously, such a condition is not satisfied for $\left(\phi_{\alpha}, \phi_{\beta}\right)=$ $(0,0)$ (which is natural because an induction motor is not controllable in the absence of flux), and at the minimum of $W\left(\phi_{\alpha}, \phi_{\beta}, \theta\right)$, so that the hypotheses of Theorem 2 are not satisfied. Therefore, we have to make use of Theorem 3. The change of relative degree at $\left(\phi_{\alpha}, \phi_{\beta}\right)=(0,0)$ is structural to the problem. The incidence of the minimum of $W$ can be reduced by imposing it in $\left(\phi_{\alpha}, \phi_{\beta}\right)=(0,0)$. If $W$ is chosen such that $\frac{\partial W}{\partial \phi_{\alpha}} \phi_{\alpha}+\frac{\partial W}{\partial \phi_{\beta}} \phi_{\beta}=0$ only at the origin, this function is negative everywhere except the origin or positive everywhere except the origin (by continuity). In fact, only the latter is possible when we consider positive definite functions $W$ with a minimum at the origin. Condition (17) then reduces to

$$
\frac{\partial W}{\partial \phi_{\alpha}} \phi_{\alpha}+\frac{\partial W}{\partial \phi_{\beta}} \phi_{\beta}>0
$$

for all $\left(\phi_{\alpha}, \phi_{\beta}\right) \neq(0,0)$. 
Remark 2 Condition (18) amounts to having the image of the function $W\left(\phi_{\alpha}, \phi_{\beta}, \theta\right)$ on any straight line going through the origin in the plane $\left(\phi_{\alpha}, \phi_{\beta}\right)$ "like a parabola", with its minimum at the origin. Any positive definite polynomial, which is the sum of homogeneous positive semidefinite polynomials, satisfies (18). Indeed, let

$$
W\left(\phi_{\alpha}, \phi_{\beta}\right)=\sum_{i=1}^{q} P_{i}\left(\phi_{\alpha}, \phi_{\beta}\right)
$$

with $P_{i}$ a positive semidefinite homogeneous polynomial of order $p_{i} \in \mathbb{N _ { 0 }}$. It can easily be seen that

$$
\begin{aligned}
\frac{\partial W}{\partial \phi_{\alpha}} \phi_{\alpha}+\frac{\partial W}{\partial \phi_{\beta}} \phi_{\beta} & =\sum_{i=1}^{q} p_{i} P_{i}\left(\phi_{\alpha}, \phi_{\beta}\right) \\
& =p W\left(\phi_{\alpha}, \phi_{\beta}\right)+\sum_{i=1}^{q}\left(p_{i}-p\right) P_{i}\left(\phi_{\alpha}, \phi_{\beta}\right) \\
& \geq p W\left(\phi_{\alpha}, \phi_{\beta}\right) \\
& >0 \quad \text { when }\left(\phi_{\alpha}, \phi_{\beta}\right) \neq(0,0)
\end{aligned}
$$

where $p=\min _{i} p_{i}$.

The linear part of system (15) with the linearizing feedback is in the form

$$
\left\{\begin{array}{l}
\dot{\xi}_{1}=\xi_{2} \\
\dot{\xi}_{2}=v_{1} \\
\dot{\xi}_{3}=v_{2}
\end{array}\right.
$$

with $\xi_{1}=y_{1}, \xi_{2}=\dot{y}_{1}=c\left(I_{\beta} \phi_{\alpha}-I_{\alpha} \phi_{\beta}\right), \xi_{3}=y_{2}$. We write the rest of the system, that is the zero dynamics and the interconnection, on the basis of the $\left(\phi_{\alpha}, \phi_{\beta}\right)$ subsystem of the original system (15). Because the original interconnection is made through the current variables, while it should be made through the $\xi$ variables to obtain a normal form, we need to substitute those current variables in the expression of $\left(\dot{\phi}_{\alpha}, \dot{\phi}_{\beta}\right)$. In order to do so, we reconsider the equalities

$$
\begin{aligned}
\xi_{2}= & c\left(I_{\beta} \phi_{\alpha}-I_{\alpha} \phi_{\beta}\right) \\
\xi_{3}= & \frac{\partial W}{\partial \phi_{\alpha}}\left(-a \phi_{\alpha}-v\left(\xi_{1}+\omega_{0}\right) \phi_{\beta}+b I_{\alpha}\right) \\
& +\frac{\partial W}{\partial \phi_{\beta}}\left(-a \phi_{\beta}+v\left(\xi_{1}+\omega_{0}\right) \phi_{\alpha}+b I_{\beta}\right)+\nu\left(W\left(\phi_{\alpha}, \phi_{\beta}, \theta\right)-L\right)
\end{aligned}
$$

which can be inverted to obtain the currents as functions of $\xi$ and $\phi$ and which, injected into the $\left(\phi_{\alpha}, \phi_{\beta}\right)$ subsystem of (15), yields:

$$
\begin{aligned}
& \dot{\phi}_{\alpha}=\frac{1}{c\left(\frac{\partial W}{\partial \phi_{\alpha}} \phi_{\alpha}+\frac{\partial W}{\partial \phi_{\beta}} \phi_{\beta}\right)} {\left[-c v\left(\xi_{1}+\omega_{0}\right)\left(\phi_{\alpha}^{2}+\phi_{\beta}^{2}\right) \frac{\partial W}{\partial \phi_{\beta}}\right.} \\
&\left.-c \phi_{\alpha} \nu\left(W\left(\phi_{\alpha}, \phi_{\beta}, \theta\right)-L\right)-b \frac{\partial W}{\partial \phi_{\beta}} \xi_{2}+c \phi_{\alpha} \xi_{3}\right] \\
& \dot{\phi}_{\beta}=\frac{1}{c\left(\frac{\partial W}{\partial \phi_{\alpha}} \phi_{\alpha}+\frac{\partial W}{\partial \phi_{\beta}} \phi_{\beta}\right)}\left[c v\left(\xi_{1}+\omega_{0}\right)\left(\phi_{\alpha}^{2}+\phi_{\beta}^{2}\right) \frac{\partial W}{\partial \phi_{\alpha}}\right. \\
&\left.-c \phi_{\beta} \nu\left(W\left(\phi_{\alpha}, \phi_{\beta}, \theta\right)-L\right)+b \frac{\partial W}{\partial \phi_{\alpha}} \xi_{2}+c \phi_{\beta} \xi_{3}\right]
\end{aligned}
$$


Theorem 3 can be applied in this case. Indeed, the zero dynamics

$$
\begin{aligned}
& \dot{\phi}_{\alpha}=\frac{1}{c\left(\frac{\partial W}{\partial \phi_{\alpha}} \phi_{\alpha}+\frac{\partial W}{\partial \phi_{\beta}} \phi_{\beta}\right)}\left[-c v \omega_{0}\left(\phi_{\alpha}^{2}+\phi_{\beta}^{2}\right) \frac{\partial W}{\partial \phi_{\beta}}-c \phi_{\alpha} \nu\left(W\left(\phi_{\alpha}, \phi_{\beta}, \theta\right)-L\right)\right] \\
& \dot{\phi}_{\beta}=\frac{1}{c\left(\frac{\partial W}{\partial \phi_{\alpha}} \phi_{\alpha}+\frac{\partial W}{\partial \phi_{\beta}} \phi_{\beta}\right)}\left[c v \omega_{0}\left(\phi_{\alpha}^{2}+\phi_{\beta}^{2}\right) \frac{\partial W}{\partial \phi_{\alpha}}-c \phi_{\beta} \nu\left(W\left(\phi_{\alpha}, \phi_{\beta}, \theta\right)-L\right)\right]
\end{aligned}
$$

yield global convergence to the closed curve $\gamma \equiv W\left(\phi_{\alpha}, \phi_{\beta}, \theta\right)=L$ (except if the initial condition is at the origin, where the dynamics are not defined) since

$$
\dot{W}\left(\phi_{\alpha}, \phi_{\beta}, \theta\right)=-\nu\left(W\left(\phi_{\alpha}, \phi_{\beta}, \theta\right)-L\right)
$$

If $c v \omega_{0}\left(\phi_{\alpha}^{2}+\phi_{\beta}^{2}\right) \neq 0$ when $W\left(\phi_{\alpha}, \phi_{\beta}, \theta\right)=L$, there is no equilibrium on $\gamma$ (this is the case when $\omega_{0} \neq 0$ ). Therefore, $\gamma$ is an almost globally attractive limit cycle.

In order to apply Theorem 3 and have $\Gamma$ be an almost globally attractive limit cycle for the whole system, Assumptions 1, 2, and 3 need to be satisfied:

- It can easily be checked that the linearity condition of Assumption 1 is satisfied when $W$ is a positive definite polynomial, sum of positive semidefinite polynomials. Indeed, there exists $\eta_{1}, \eta_{2}>0$ constants such that

$$
\left\|\left(\begin{array}{c}
\frac{-c v \xi_{1}\left(\phi_{\alpha}^{2}+\phi_{\beta}^{2}\right) \frac{\partial W}{\partial \phi_{\beta}}-b \frac{\partial W}{\partial \phi_{\beta}} \xi_{2}+c \phi_{\alpha} \xi_{3}}{c\left(\frac{\partial W}{\partial \phi_{\alpha}} \phi_{\alpha}+\frac{\partial W}{\partial \phi_{\beta}} \phi_{\beta}\right)} \\
\frac{c v \xi_{1}\left(\phi_{\alpha}^{2}+\phi_{\beta}^{2}\right) \frac{\partial W}{\partial \phi_{\beta}}+b \frac{\partial W}{\partial \phi_{\alpha}} \xi_{2}+c \phi_{\beta} \xi_{3}}{c\left(\frac{\partial W}{\partial \phi_{\alpha}} \phi_{\alpha}+\frac{\partial W}{\partial \phi_{\beta}} \phi_{\beta}\right)}
\end{array}\right)\right\| \leq \eta_{1}\|\xi\|\left\|\left(\begin{array}{c}
\phi_{\alpha} \\
\phi_{\beta}
\end{array}\right)\right\|+\eta_{2}\|\xi\|
$$

- Assumption 2 is satisfied with $W\left(\phi_{\alpha}, \phi_{\beta}, \theta\right)$ because of (19) and because $W$ is polynomial.

- Assumption 3 is verified because

$$
L_{f(z)+\psi(z, \xi)}=-\nu\left(W\left(\phi_{\alpha}, \phi_{\beta}, \theta\right)-L\right)+\xi_{3}
$$

For any initial condition, $\xi_{3}(t)$ is bounded, so that $\xi_{3}$ belongs to an interval (with $B$ as lower bound). We can then take $\delta>0$ such that $L_{f(z)+\psi(z, \xi)}>0$ for $W\left(\phi_{\alpha}, \phi_{\beta}, \theta\right) \leq \delta$. Indeed, $\lim _{s \rightarrow-L} \nu(s)=-\infty$, implies that, for $W$ small enough, there is $\nu\left(W\left(\phi_{\alpha}, \phi_{\beta}, \theta\right)-L\right)<B$.

In order to illustrate this example, we present some simulations. The parameters of the simulated motor are those of the motor of the L.A.G ([10]). The target angular velocity is the nominal angular velocity of that motor: 
$150 \mathrm{rad} / \mathrm{s}$. We have chosen to create a limit cycle using for $W$ a positive definite polynomial, sum of homogeneous positive semidefinite polynomials, as suggested in Remark 2:

$$
W\left(\phi_{\alpha}, \phi_{\beta}\right)=\left(\phi_{\alpha}^{2}+\phi_{\beta}^{2}\right)+5000\left(\phi_{\alpha}^{2}-\phi_{\beta}^{2}\right)^{2} \phi_{\alpha}^{2} \phi_{\beta}^{2}
$$

and $L=0.001$. Regulating the outputs, this results in a limit cycle in the $\left(\phi_{\alpha}, \phi_{\beta}\right)$ plane having an octogone-like shape (see Figure 3$)$. The convergence of $\omega$ and $W$ towards their set-points is shown in the first two figures of Figure 4. The other two represent the evolution of $\phi_{\alpha}$ and $I_{\alpha}$ once $W$ and $\omega$ have reached their target. The time-span for those last two figures is a period of the mechanical rotation of the motor $\left(\frac{2 \pi}{150}\right)$. From those figures, we see that the flux states oscillations look like sinusoids, except at the peaks where the nonlinearities can be noticed. On the other hand, the current states oscillate much more.

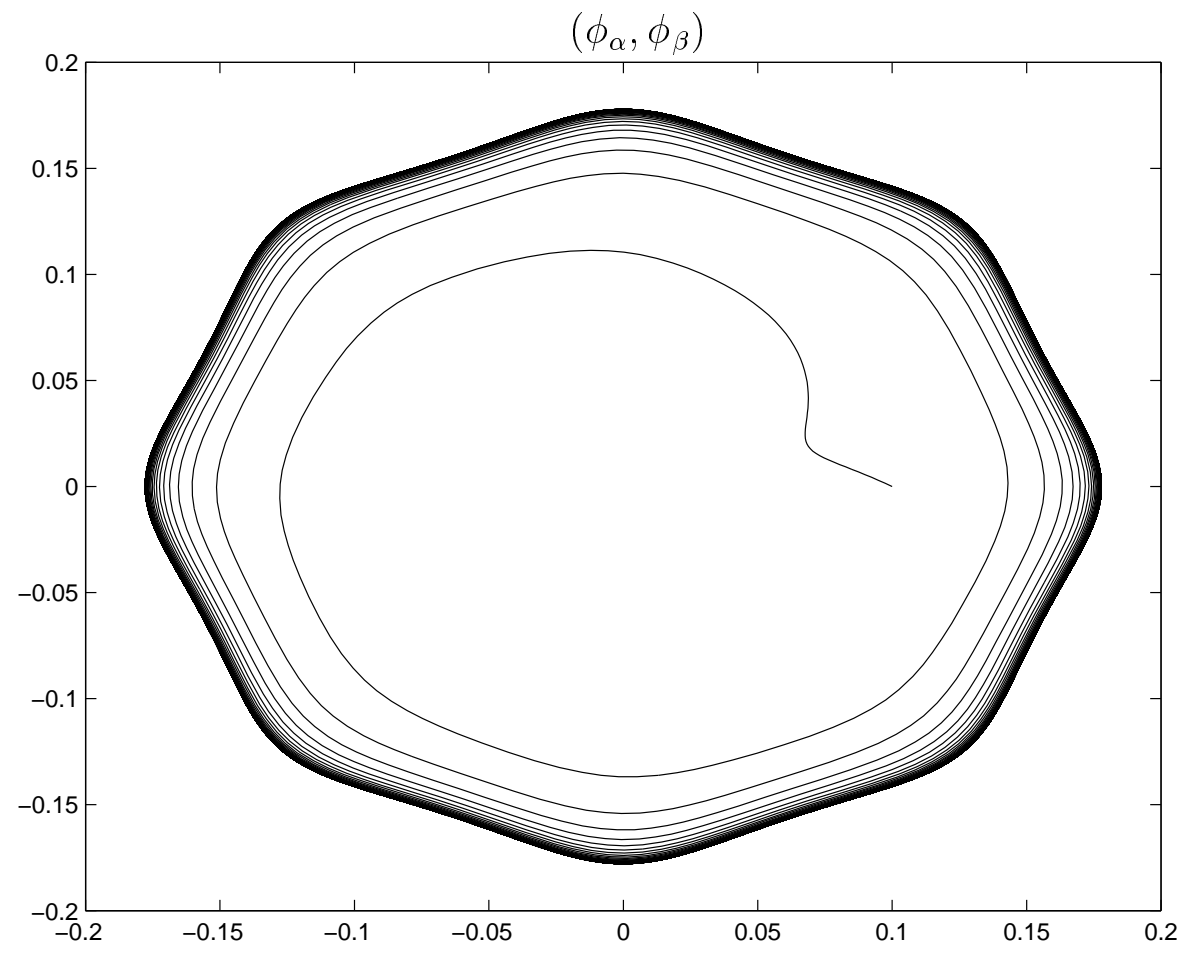

Figure 3: Limit cycle in the phase plane of the $\left(\phi_{\alpha}, \phi_{\beta}\right)$ states 

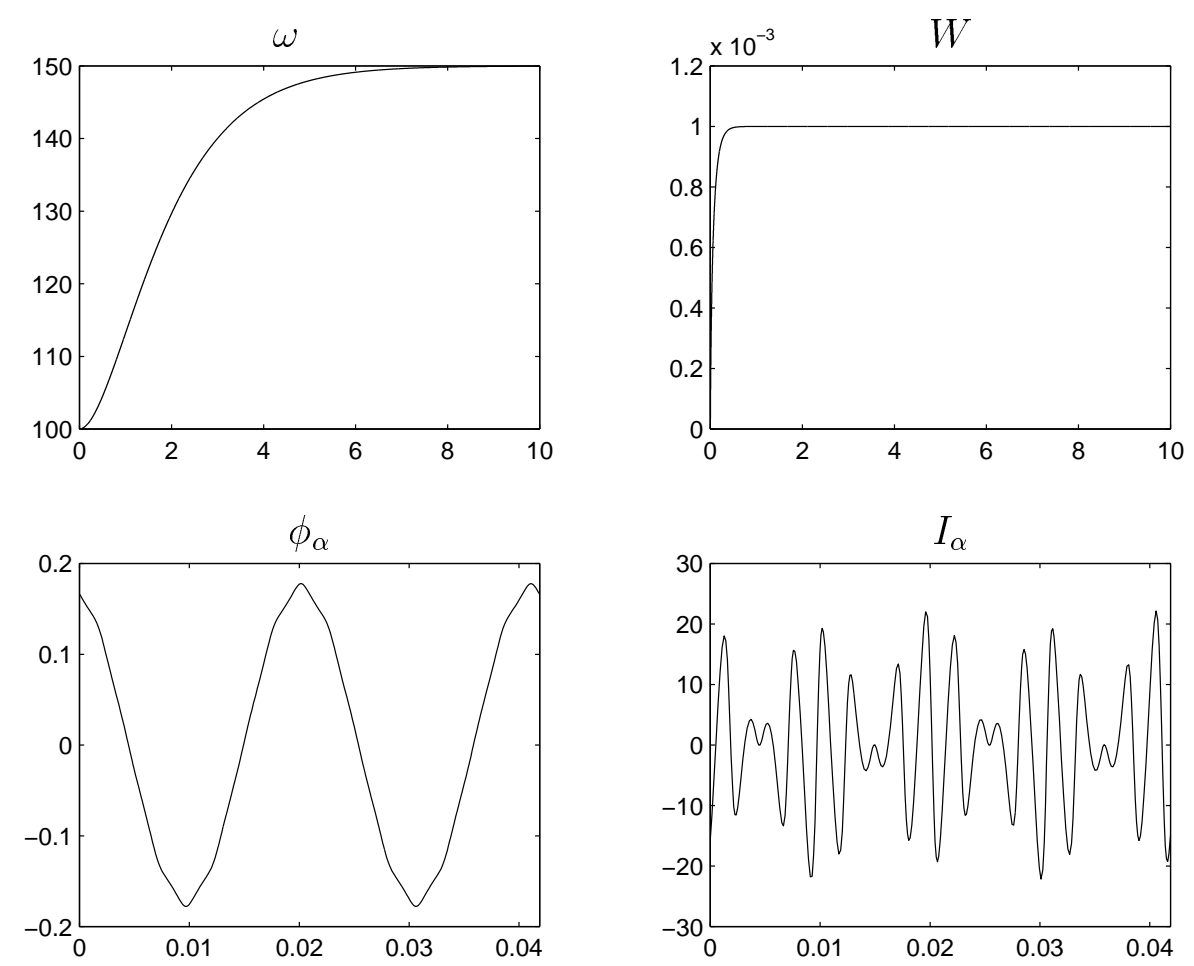

Figure 4: Time evolution of the outputs and the states of the induction motor

\section{Conclusion}

In this paper, we have presented two results about the existence of an almost globally attractive limit cycle in a nonlinear system when there is an almost globally attractive limit cycle in the zero-dynamics. Those results are then used to present a methodology for the design of limit cycles in systems having more inputs available than outputs to regulate. Finally, this methodology is illustrated on the induction motor, where the presence of a limit cycle is somewhat natural. It has also been applied to the orbital stabilization of underactuated mechanical systems in [3].

\section{Acknowledgments}

This research is supported by the NACO2 project of the Research Training Network of the European Commission (reference: HPRN-CT-1999-00046). We wish to thank the anonymous reviewers for their constructive comments. 


\section{References}

[1] Besançon G., "Contribution à l'étude et à l'observation des systèmes non linéaires avec recours au calcul formel," PhD. Thesis, Grenoble, 1996.

[2] Canudas-de-Wit C., Ramirez J., "Optimal torque control for current-fed induction motors," IEEE Trans. Automat. Control 44, no. 5, 1084-1089, 1999.

[3] Canudas de Wit C., Espiau B., Urrea C., "Orbital stabilization of underactuated mechanical systems," to be presented at IFAC02, Barcelona, Spain.

[4] De Luca A., Ulivi G., "Design of an Exact Nonlinear Controller for Induction Motors," IEEE Trans. Automat. Control 34, no. 12, 13041307, 1989.

[5] Guckenheimer J., Holmes P., "Nonlinear oscillations, dynamical systems, and bifurcations of vector fields," Springer Verlag, New York, 1983.

[6] Hauser J., Choo Chung C., "Converse Lyapunov functions for exponentially stable periodic orbits," Systems \& Control Letters, 23, 27-34, 1994.

[7] Isidori A., "Nonlinear Control Systems," 2nd. ed., Springer-Verlag, Berlin, 1989.

[8] Khalil H., "Nonlinear Systems," 3rd ed., Prentice Hall, 2002.

[9] Marconi L., Isidori A., Sarrani A., "Autonomous vertical landing on an oscillating platform: an internal-model based approach," Automatica 38, no. 1, 21-32, 2002.

[10] Ramirez J., "Contribution à la commande optimale de machines asynchrones," PhD. Thesis, Grenoble, 1998.

[11] Sepulchre R., Jankovic M., Kokotovic P., "Constructive Nonlinear Control", Springer Verlag, 1997. 